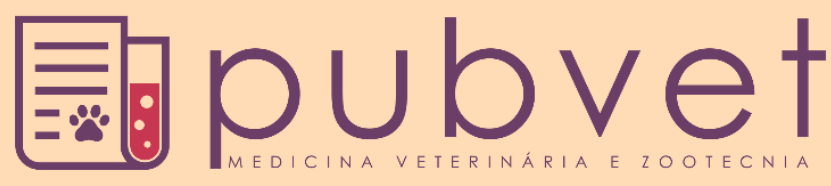

https://doi.org/10.31533/pubvet.v14n10a668.1-6

\title{
Estudo epidemiológico da erliquiose monocítica canina na cidade de Barreiras - Bahia
}

\author{
Ana Manuella Souza de Babo', Alanna Machado ${ }^{2}$, Emesson Bastos ${ }^{2}$, Rodrigo de Lima Carneiro ${ }^{3}$, \\ Rosane dos Santos², Saady Cézar²*, Wilker Souza ${ }^{2}$ \\ ${ }^{I}$ Professora do curso de Medicina Veterinária da Unidade Regional Brasileira de Educação UNIRB. Barreiras-BA. \\ ${ }^{2}$ Acadêmica do curso de Medicina Veterinária da Unidade Regional Brasileira de Educação UNIRB. Barreiras-BA. \\ ${ }^{3}$ Professor do curso de Medicina Veterinária da Universidade Estadual da Bahia UNEB. Barreiras-BA \\ *Autor para correspondência, E-mail: cezarsaady@gmail.com
}

\begin{abstract}
Resumo. Considerada um agravo a saúde por se tratar de uma zoonose e de grande importância na rotina clínica médica de pequenos animais, a Erliquiose Monocítica Canina pode causar febre e diarreia, além de desordens no sistema neurológico e renal. Sendo assim, tendo em vista a relevância clínica da patologia, objetivou-se identificar o números de casos ocorridos na cidade de Barreiras, Oeste da Bahia, durante o período de Janeiro a Dezembro de 2018, relacionando-os a condições climáticas presentes em determinados intervalos, raças em que a incidência de casos se manifestou de uma forma mais eminente, além do sexo e idade dos cães acometidos, estabelecendo assim, diferentes causas. Foi constatado a partir de um estudo epidemiológico, mais de 140 diagnósticos, com superior acometimento durante o período chuvoso, de fêmeas sem raça definida, entre 1 a 5 anos de idade. É imprescindível nos atentarmos ao que diz respeito a profilaxia, visando diminuir o número de casos e intensificar o bem-estar animal.
\end{abstract}

Palavras chave: hemoparasitose, ixodidiose, rickettsiose

\section{Epidemiological study of prevalence of canine monocytic erliquiosis in the city of Barreiras - Bahia}

\begin{abstract}
Considered a health problem because it is a zoonosis and of great importance in the medical clinical routine of small animals, Canine Monocytic Erlichiosis can cause fever and diarrhea, in addition to disorders in the neurological and renal system. Thus, in view of the clinical relevance of the pathology, the objective was to identify the number of cases that occurrend in the city of Barreiras Western Bahia, during the period from January to December 2018, relating them to climatic conditions presente in certain intervals, breeds in which the incidence of cases was manifested in a more prominent way, in addition to the sex and age of the affected dogs, thus establishing diferente causes. It was found from na epidemiological study, more than 140 diagnoses, with superior involvement during the rainy season off emales, without defined race, between 1 to 5 years of age. It is essential to pay attention to prophylaxis, in order to reduce the number of cases and intensify animal welfare.
\end{abstract}

Keywords: hemoparasitosis, ixodidiosis, rickettsiosis

\section{Estudio epidemiológico de prevalência de la erliquiosis monocítica canina em la ciudad de Barreiras - Bahia}

Resumen. Considerada un problema de salud porque es una zoonosis y de gran importancia em la rutina clínica médica de animales pequeños, la Erlichiosis Monocítica Canina puede causar fiebre y diarrea, además de trastornos en el sistema neurológico y renal. Por lo tanto, 
em vista de la relevancia clínica de la patologia, el objetivo fue identificar el número de casos que ocurrieron en la ciudad de Barreiras Bahia Occindental, durante el período de enero a diciembre de 2018, relacionándolos con las condiciones climáticas presentes em ciertos intervalos, razas en las cuales la incidencia de casos se manifestó de manera más prominente, además del sexo y la edad de los perros afectados estableciendo así diferentes causas. Se encontró en un estudio epidemiológico, más de 140 diagnósticos, con una participación superior durante la estación lluviosa de hembras mestizas con edad entre 1 y 5 años. Es esencial prestar atención a la profilaxis para reducir el número de casos e intensificar el bienestar animal.

Palabras clave: hemoparasitosis, ixodidiosis, rickettisiosis

\section{Introdução}

A Erliquiose canina é uma doença emergente e infectocontagiosa de alta incidência na rotina clínica veterinária, causando sérios prejuízos à saúde animal e indiretamente à saúde humana, por se tratar de uma zoonose (Isola et al., 2012; Machado, 2004; Silva et al., 2013). A doença é caracterizada por manifestações clínicas multissistêmicas, que variam na intensidade de acordo com as fases da doença: aguda, subclínica ou crônica. Os sinais clínicos mais frequentemente observados são apatia, inapetência, palidez de mucosas, febre, linfadenopatia, esplenomegalia, hemorragias e uveíte (Nakaghi et al., 2008).

Analisando a propagação do Erlichia canis no Rhipicephalus sanguineus, Bremer et al. (2005) e Ferreira et al. (2012) discorrem que a riquétsia "se multiplica nas células epiteliais do intestino, hemócitos e na glândula salivar", ocorrendo assim a transmissão transestadial, permitindo que o carrapato permaneça infectado durante toda a sua vida e várias gerações ou intraestadial (o macho adulto se infecta em um cão portador e transmite para outro cão sensível).

Com relação a prevalência, Labarthe et al. (2003) apontam que a doença é observada com mais frequência na região Nordeste e Centro-Oeste e em menores densidades em climas temperados, como por exemplo no Paraná. Vale ressaltar que a incidência da doença é mais comum nos meses mais quentes quando há um maior desenvolvimento do carrapato, podendo ser diagnosticada durante todo o ano; não existe uma predileção etária para o E. canis. "A maior prevalência observada é na região Nordeste (43\%) e a menor na região Sul do país (1,70\%)" (Teixeira et al., 2010).

A infecção por E. canis é relatada mundialmente sendo mais comumente encontrada nas regiões tropicais e subtropicais do mundo (Baneth, 2006; Greene, 2006). A Erliquiose Monocítica Canina é frequentemente diagnosticada acometendo cães na América do Norte e América do Sul (Tzipory et al., 2010).

Existem várias causas que podem estar intimamente ligadas a severidade da doença, entre elas a idade do animal, suscetibilidade racial, alimentação, virulência da cepa infectante e possíveis doenças concomitantes (Silva et al., 2010). Em relação a isso, Tilley \& Smith (2015) apontam que a patologia aparenta ser mais graves em cães da raça Dobermanns, Pinscher e Pastor Alemão.

\section{Material e métodos}

Definida a problemática da pesquisa em conjunto com a finalidade, foi elaborado alternativas para a coleta de dados, análise e interpretação. O tipo de estudo utilizado é de base epidemiológica descritiva, buscando a descrição numérica de amostra representativa e posteriormente o estabelecimento da casuística e comparação em relação aos pontos chaves pertencentes ao propósito no qual se almeja de forma fidedigna.

Os dados, informações e materiais necessários a pesquisa foram coletados no Laboratório Animal Lab, localizado na rua Alberto Coimbra, bairro Sandra Regina, anexado a CLIMEV Clínica Veterinária, na cidade de Barreiras, Oeste da Bahia.

Foram realizadas duas visitas, ambas na primeira semana do mês de maio, para a devida coleta de dados, em que o responsável técnico pelo laboratório, o médico veterinário André Carloto Vielmo, CRMV-BA 2356, nos cedeu diversas planilhas com as casuísticas já determinadas antes da coleta de dados. Vale ressaltar que os diagnósticos foram feitos através da técnica de esfregaço sanguíneo realizados no ano de 2018. 
Além da procura por número gerais, foi realizado pelo roteiro norteador, a coleta de dados acerca de diferentes casuísticas, sendo elas: mês exato do diagnóstico, gênero do cão, seja ele masculino ou feminino, além de idade e raça.

Portanto, a partir do conjunto de informações e números cedidos pelo responsável técnico do laboratório citado, foi possível estabelecer estatísticas bem como bases epidemiológicas do acometimento de cães por Erliquiose Monocítica Canina na cidade de Barreiras Bahia.

A epidemiologia moderna permite a interação com a estatística a partir da coleta e análise de dados, que inclusive é representada por meio de gráficos, que serão usualmente utilizados no presente projeto, extraindo informações diretamente da realidade do objeto de estudo.

\section{Resultados e discussão}

Foram diagnosticados 143 cães acometidos pela bactéria Erlichia canis durante o período de janeiro a dezembro do ano de 2018 no laboratório Animal Lab, na cidade de Barreiras-BA. No gráfico a seguir há a representação desses casos paralelamente relacionados aos meses em que foram identificados.

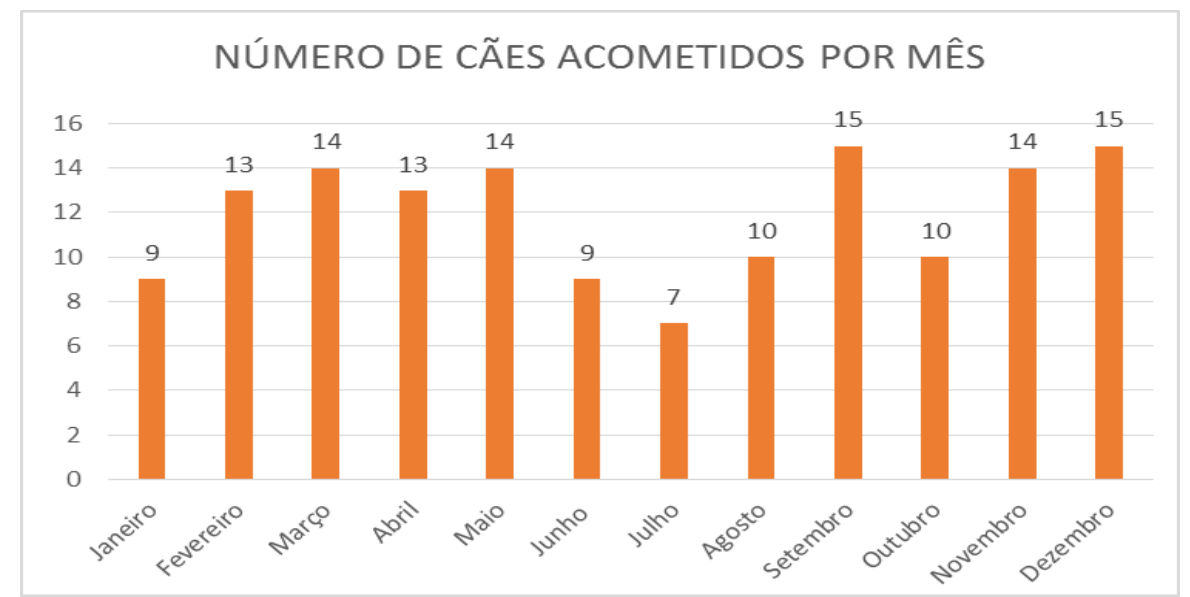

Gráfico 1. Número de cães diagnosticados com Erliquiose Monocítica Canina em Barreiras, Bahia.

Observou-se que dos 143 cães diagnosticados com Erliquiose, 82 eram fêmeas (57\%) e 61 machos (43\%). Conforme expresso no gráfico 2, há uma superioridade na afecção de fêmeas. Este resultado confirma os já obtidos na literatura nacional, em que $61 \%$ dos cães acometidos pela doença eram fêmeas (Moreira et al., 2003). Porém não existe uma casuística referente ao gênero de cães no Brasil, se há uma prevalência maior de fêmeas ou machos, portanto, nada se pode afirmar acerca do número obtido.

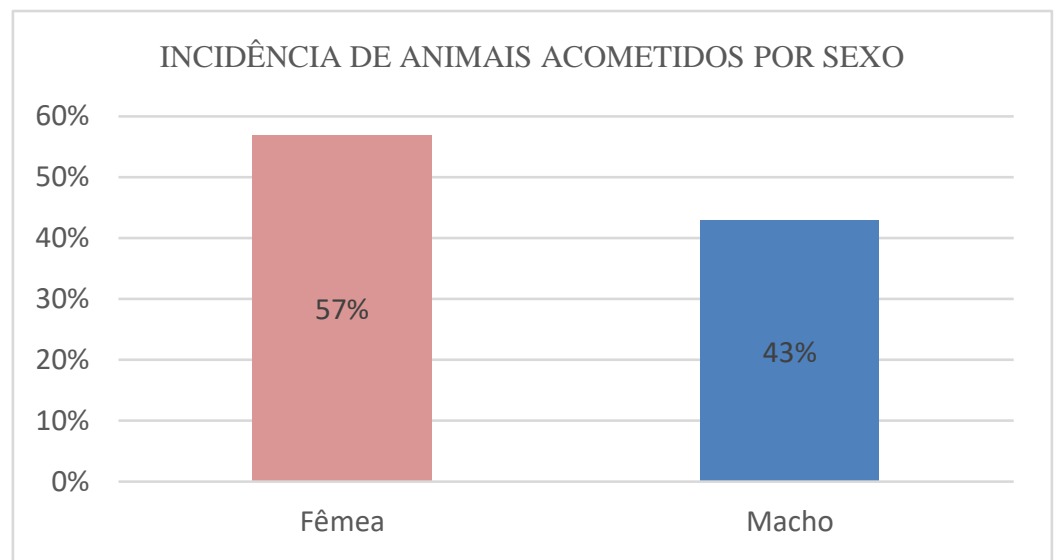

Gráfico 2. Incidência de animais acometidos por sexo na cidade de Barreiras, Bahia no ano de 2018.

Com relação a faixa etária dos cães com resultado positivo, observou-se que dos 143 utilizados na amostragem, 37 tinham menos de 1 ano; 49 tinham entre 1 a 5 anos de idade; 12 apresentavam entre 6 e 9 anos, e outros 12 tinham mais de 10 anos de idade, considerando-se uma idade bem avançada. Nos 
dados, 33 constavam como "não identificado", provavelmente porque as clínicas solicitantes não anexaram esses dados na solicitação do exame ou pelo simples fato de que o proprietário ou o tutor do animal não ter conhecimento a respeito dessa questão.

Em porcentagem, temos os seguintes dados referentes a faixa etária dos animais diagnosticados com Erlichia canis (ressaltando que os valores estão apresentados em forma arredondada, porém sem deturpar a legitimidade do presente estudo).

Sendo assim, 34\% tem entre 1 a 5 anos; $27 \%$ menos de um ano; $23 \%$ não foram identificados; $8 \%$ entre 6 e 9 anos e $8 \%$ com mais de 10 anos.

Considerando que em $23 \%$ dos casos não foram identificados ao qual grupo de faixa etária o animal pertence, coletamos assim novos dados a respeito dos quatro grupos, considerando uma escala de 0 a $100 \%$ absolutos, com a exclusão dos não intitulados N.I.

Observou-se que aproximadamente $34 \%$ apresentam menos de 1 anos; $45 \%$ tem entre 1 e 5 anos; $11 \%$ entre 6 e 7 anos e $11 \%$ com mais de 10 anos de idade (Gráfico 3).

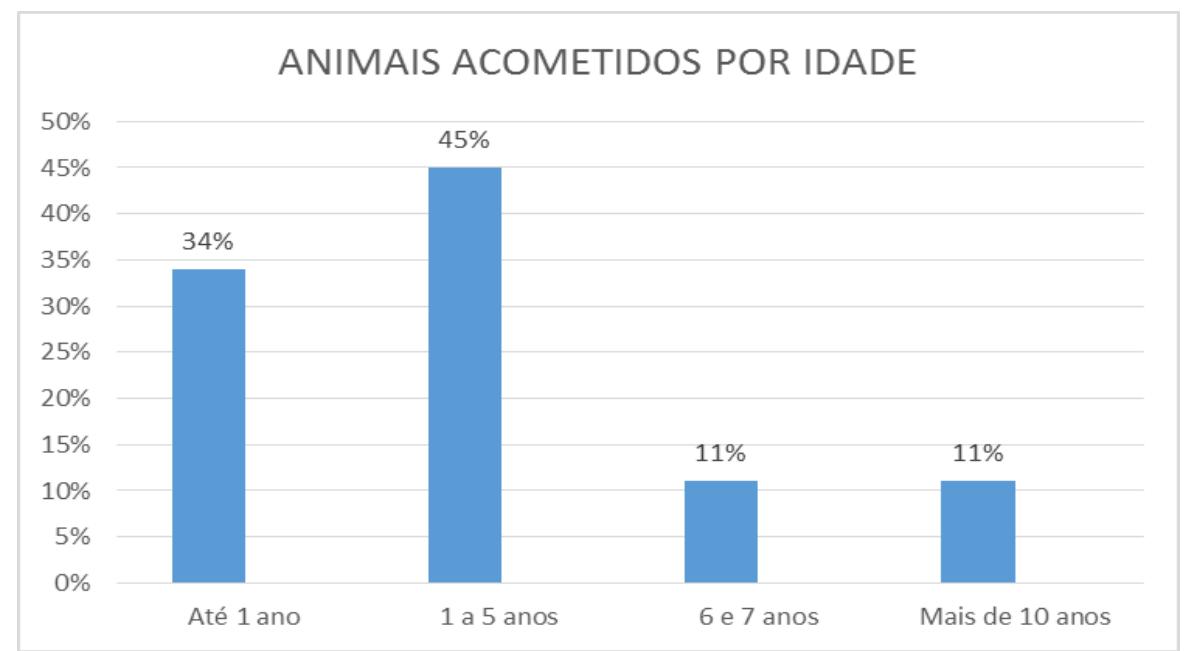

Gráfico 3. Faixa etária dos animais acometidos por Erliquiose Monocítica Canina na cidade de Barreiras, Bahia, no ano de 2018.

Em avaliação quanto ao acometimento de raças, os pertencentes ao grupo SRD (sem raça definida), compôs a progênie em que a incidência de diagnóstico positivo ocorreu com maior frequência, com um total de 38 casos; seguida da linhagem Shih Tzu, com 19 casos e Poodle com 16; Pinscher com 9 e Pastor Alemão 7; Rottweiller, Basset e Pitbull com 5 casos cada; Yorkshire e Husk Siberiano com 4 casos cada; Pug, Boxer, Sharpei, Pointer e Lhasa Apso, apresentaram 3 cães acometidos cada; Labrador com 2 e todas as seguintes raças manifestaram apenas 1 cão com Erliquiose durante todo o ano de 2018, são elas: Boder Colie, American Staffords, Golden, Cocker Spaniel, Spitz, Bulldog, Daschgund e Chiuhahua.

Isso nos mostra que aproximadamente $27 \%$ dos cães com Erlichia canis não tinham raça definida, 13\% eram Shih Tzu, 11\% Poodle, 6\% Pinscher, e 7\% Pastor Alemão (Gráfico 4).

O clima na cidade de Barreiras-BA é caracterizado por uma estação seca (maio a setembro) e outra chuvosa (outubro a abril). Durante o período seco, foi observado uma média de 11 casos por mês. Já no período chuvoso, foi encontrado a dizima periódica de 14,7 em média de casos por mês, ou seja, notouse um discreto aumento no número de casos diagnosticados de Erlichiose no período chuvoso na cidade.

O número de casos positivos está diretamente associado à distribuição do vetor encontrado principalmente em regiões de clima tropical. Essa diferença pode ser acarretada pela maior ou menor quantidade de carrapatos, a origem dos animais, a sensibilidade dos diferentes testes e o número de casos utilizados no estudo (Carlos et al., 2007; Rodriguez-Vivas et al., 2005).

Esta relação é confirmada por Almosny (1998), tal que o autor cita que a Erlichiose sofre influência sazonal referente a prevalência do seu vetor. 
Um estudo realizado por Borin et al. (2009) revelou que a ocorrência da Erlichiose canina em fêmeas foi significativamente mais alta. Este resultado confirma os já obtidos na literatura nacional em que 61,5\% dos cães acometidos pela doença eram fêmeas (Moreira et al., 2003). Seguindo essa linha, o presente estudo destaca uma propensão maior em cães do sexo feminino, representando 82 casos entre os 143 contabilizados.

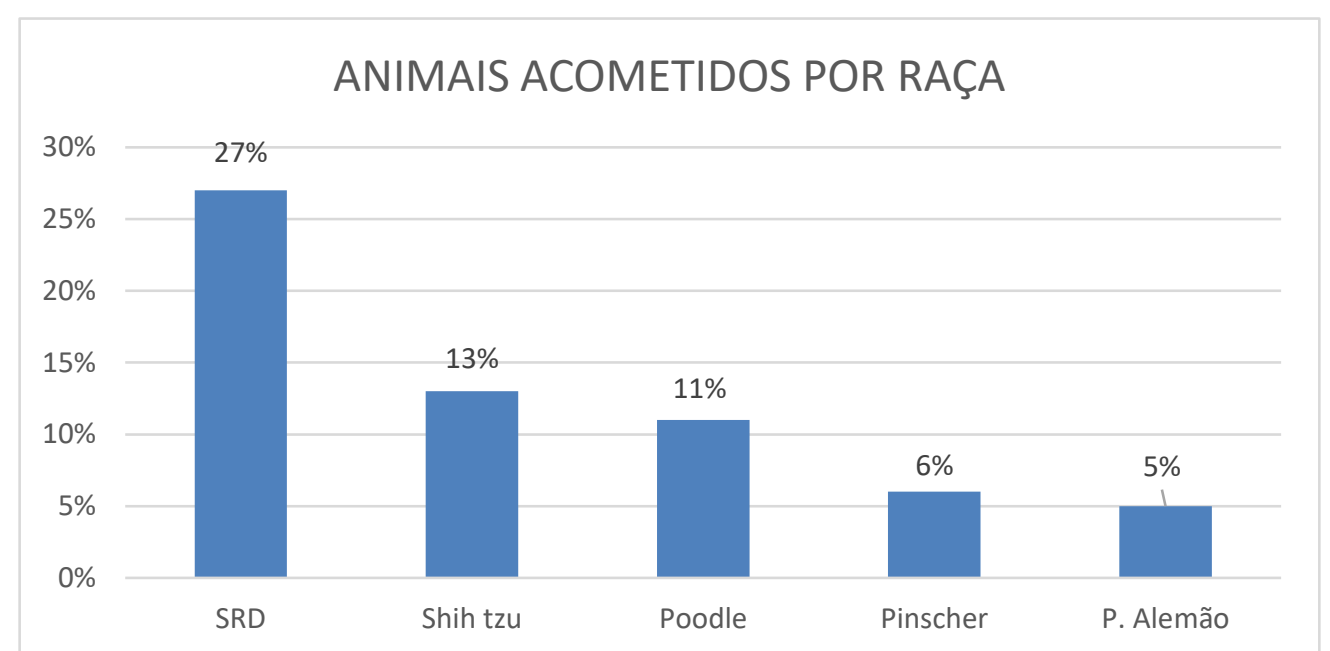

Gráfico 4. Raça dos animais acometidos por Erliquiose Monocítica Canina no ano de 2018 em Barreiras, Bahia.

Referente a faixa etária dos animais positivos, houve uma eminente prevalência em cães com até 5 anos, somando em 86 casos, considerando ainda os 33 casos em que esse dado não foi identificado, o que significa que este número provavelmente poderia ser maior. Cães acima de 5 anos representaram 24 casos. Observaram-se resultados semelhantes aos encontrados por Silva (2010) e Borin (2009), ambos evidenciando a ocorrência da enfermidade em cães com até cinco anos de idade.

Em um estudo sobre aspectos epidemiológicos, clínicos e hematológicos de 203 cães portadores da mórula de Erlichia ssp. 59\% eram de raças definidas, destacando-se Poodle 20\%, Pastor Alemão e Pinscher ambos com 13\%. Em Barreiras observou-se que em 73\% dos casos diagnosticados eram de cães com raça definida, destacando Shih Tzu com 13\% e Poodle com 11\%; os SRD registraram 27\%, isso não corrobora com o resultado do estudo citado acima, contudo vale ressaltar que nada se pode afirmar em relação a incidência vinculada a grupo racial, já que não se tem uma dimensão correta da casuística de raças.

\section{Conclusões}

Em virtude do caráter regional, e por se tratar de uma zoonose, é necessário que os profissionais em conjunto com proprietários se atentem em relação a profilaxia, com o objetivo de diminuir cada vez mais esse número alarmante na região, prevalente principalmente em períodos chuvosos, com dados ascendentes para cães entre 1 e 5 anos de idade, além de ser mais frequente em fêmeas e em animais que não apresentam raça definida.

É irrefutável a importância de novos estudos epidemiológicos para a caracterização e conhecimento da distribuição acerca das variáveis contidas neste estudo, bem como a inclusão de esfregaços sanguíneos periféricos como rotina na clínica de pequenos animais, visto que a técnica permite diagnostico rápido de diversas alterações e patologias, como a Erliquiose Monocítica Canina.

\section{Referências bibliográficas}

Almosny, N. R. P. (1998). Ehrlichia canis (Donatien \& Lestoquard, 1935): Avaliação parasitológica, hematológica e bioquímica sérica da fase aguda de cães e gatos experimentalmente infectados. Rio de Janeiro.

Baneth, G. (2006). Infectious diseases of the dog and cat. In C. E. Greene (Ed.), Hepatozzon canis Infection (3a ed., p. 698). Elsevier Health Sciences. 
Borin, S., Crivelenti, L. Z., \& Ferreira, F. A. (2009). Aspectos epidemiológicos, clínicos e hematológicos de 251 cães portadores de mórula de Ehrlichia spp. naturalmente infectados. Arquivo Brasileiro de Medicina Veterinária e Zootecnia, 61(3), 566-571. DOI: https://doi.org/10.1590/s0102-09352009000300007

Bremer, W. G., Schaefer, J. J., Wagner, E. R., Ewing, S. A., Rikihisa, Y., Needham, G. R., Jittapalapong, S., Moore, D. L., \& Stich, R. W. (2005). Transstadial and intrastadial experimental transmission of Ehrlichia canis by male Rhipicephalus sanguineus. Veterinary Parasitology, 131(1-2), 95-105. DOI: https://doi.org/10.1016/j.vetpar.2005.04.030

Carlos, R. S. A., Neta, M., Elza, S., Spagnol, F. H., Oliveira, L. L. S., Brito, R. L. L., Albuquerque, G. R., \& Almosny, N. R. P. (2007). Frequiência de anticorpos anti-Erhlichia canis, Borrelia burgdorferi e antígenos de Dirofilaria immitis em cães na microrregião Ilhéus-Itabuna, Bahia, Brasil. Revista Brasileira de Parasitologia Veterinária, 16(3), 117-120. DOI: https://doi.org/10.1590/S1984$\underline{29612007000300001}$

Ferreira, M. R. A., Freitas Filho, E. G., Dias, M., \& Moreira, C. N. (2012). Prevalência, fatores de risco e associações laboratoriais para erliquiose monocítica canina. Enciclopédia Biosfera, 8(15), 1345-1356.

Greene, C. E. (2006). Fatores ambientais de doenças infecciosas. In E. D. Ibid (Ed.), Doenças infecciosas em cães e gatos. Elsivier.

Isola, J. G. M. P., Cadioli, F. A., \& Nakage, A. P. (2012). Erliquiose canina-revisão de literatura. Revista Científica Eletrônica de Medicina Veterinária, 18, 1-11.

Labarthe, N., Campos, P. M., Barbarini, O., McKee, W., Coimbra, C. A., \& Hoskins, J. (2003). Serologic prevalence of Dirofilaria immitis, Ehrlichia canis, and Borrelia burgdorferi infections in Brazil. Veterinary Therapeutics: Research in Applied Veterinary Medicine, 4(1), 67-75.

Machado, R. Z. (2004). Erliquiose canina. Revista Brasileira de Parasitologia Veterinária, 13, 53-57.

Moreira, S. M., Bastos, C. V, Araújo, R. B., Santos, M., \& Passos, L. M. F. (2003). Retrospective study (1998-2001) on canine ehrlichiosis in Belo Horizonte, MG, Brazil. Arquivo Brasileiro de Medicina Veterinária e Zootecnia, 55(2), 141-147. DOI: https://doi.org/10.1590/s0102-09352003000200003

Nakaghi, A. C. H., Machado, R. Z., Costa, M. T., André, M. R., \& Baldani, C. D. (2008). Canine ehrlichiosis: clinical, hematological, serological and molecular aspects. Ciência Rural, 38(3), 766770. DOI: https://doi.org/10.1590/s0103-84782008000300027

Rodriguez-Vivas, R. I., Albornoz, R. E. F., \& Bolio, G. M. E. (2005). Ehrlichia canis in dogs in Yucatan, Mexico: seroprevalence, prevalence of infection and associated factors. Veterinary Parasitology, 127(1), 75-79. DOI: https://doi.org/10.1016/j.vetpar.2004.08.022

Silva, J. N., Almeida, A. do B. P. F., Boa Sorte, E. C., Freitas, A. G., Santos, L. G. F., Aguiar, D. M., \& Sousa, V. R. F. (2010). Soroprevalência de anticorpos anti-Ehrlichia canis em cães de Cuiabá, Mato Grosso. Revista Brasileira de Parasitologia Veterinária, 19(2), 108-111. DOI: https://doi.org/10.4322/rbpv.01902008

Silva, M. V. M., Fernandes, R. A., Nogueira, J. L., \& Ambrósio, C. E. (2013). Erliquiose canina: revisão de literatura. Arquivos de Ciências Veterinárias e Zoologia Da UNIPAR, 14(2), 139-143.

Teixeira, W. C., Uzêda, R. S., Gondim, L. F. P., Silva, M. I. S., Pereira, H. M., Alves, L. C., \& Faustino, M. A. G. (2010). Prevalência de anticorpos anti-Neospora caninum (Apicomplexa: Sarcocystidae) em bovinos leiteiros de propriedades rurais em três microrregiões no estado do Maranhão. Pesquisa Veterinária Brasileira, 30(9), 729-734. DOI: https://doi.org/10.1590/s0100-736x2010000900004

Tilley, P. L., \& Smith, F. K. W. (2015). Five-minute Veterinary consult: canine and feline. John Wiley $\&$ Sons.

Tzipory, N., Crawford, P. C., \& Levy, J. K. (2010). Prevalence of Dirofilaria immitis, Ehrlichia canis, and Borrelia burgdorferi in pet dogs, racing greyhounds, and shelter dogs in Florida. Veterinary Parasitology, 171(1-2), 136-139. DOI: https://doi.org/10.1016/j.vetpar.2010.03.016

Recebido: 30 de março, 2020. Aprovado: 28 de abril, 2020.

Disponível online: 6 de outubro, 2020.
Licenciamento: Este artigo é publicado na modalidade Acesso Aberto sob a licença Creative Commons Atribuição 4.0 (CC-BY 4.0), a qual permite uso irrestrito, distribuição, reprodução em qualquer meio, desde que o autor e a fonte sejam devidamente creditados 